\title{
¿Es el lactato un buen indicador de hipoxia tisular? Resultados de un estudio piloto en 21 pacientes con un traumatismo craneoencefálico
}

\author{
M.A. Merino; J. Sahuquillo*; A. Borrull; M.A. Poca*; M. Riveiro** y L. Expósito** \\ Unidad de Investigación de Neurotraumatología y Neurocirugía (UNINN). *Servicio de Neurocirugía. **Unidad de Cuidados Intensivos de \\ Neurotraumatología. Hospital Universitario Vall d'Hebron, Barcelona. Institut Fundació de Recerca Vall d'Hebron. Universitat Autònoma de \\ Barcelona.
}

\section{Resumen}

El lactato y el índice lactato-piruvato (LP) son dos marcadores utilizados para la detección de la hipoxia cerebral en pacientes que han sufrido un traumatismo craneoencefálico (TCE). Estos dos marcadores presentan un comportamiento más complejo de lo que se esperaría debido a que pueden estar anormalmente elevados en circunstancias que no cursan con hipoxia tisular detectable. Este comportamiento debe ser considerado en el diagnóstico diferencial puesto que refleja también una alteración del metabolismo energético cerebral.

Objetivos. 1. Describir las características del metabolismo energético cerebral que se observa en la fase aguda de los pacientes que han sufrido un TCE en base a los dos indicadores tradicionales del metabolismo anaeróbico: lactato e índice LP, 2. Determinar la concordancia entre ambos indicadores para clasificar la incidencia del metabolismo anaeróbico y 3. Clasificar los diferentes tipos de alteración metabólica que se observa en los pacientes con un TCE moderado o grave en base a estos dos indicadores.

Material y métodos. Se seleccionaron aleatoriamente veintiún pacientes de una cohorte de pacientes con TCE moderado o grave admitidos en la unidad de cuidados intensivos y monitorizados mediante microdiálisis (MD) cerebral y presión tisular de oxígeno $\left(\mathrm{PtiO}_{2}\right)$. Se analizaron los niveles de lactato e índice LP de cada microvial generado en las primeras 96 horas tras el TCE. Estos datos fueron correlacionados con los valores de $\mathrm{PtiO}_{2}$.

Resultados. El lactato y el índice LP estuvieron elevados el $49,5 \%$ y el $38,4 \%$ del tiempo total monitorizado respectivamente, siendo su incidencia y comportamiento muy heterogéneos entre los pacientes. La concordancia entre estos dos biomarcadores a la hora de determinar episodios de disfunción metabólica fue muy débil (K =0,29; IC 95\%: 0,24-

Recibido: 10-09-09. Aceptado: 10-02-10
0,34). En base a los niveles de lactato e índice LP se

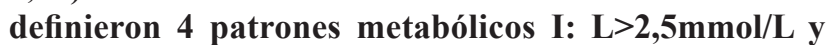
LP>25; II: $L>2,5 \mathrm{mmol} / \mathrm{L}$ y $L P \leq 25$; III: $L \leq 2,5 \mathrm{mmol} / \mathrm{L}$ y $L P \leq 25$; IV: $L \leq 2,5 \mathrm{mmol} / \mathrm{L}$ y $L P>25$. Los niveles de oxígeno se encontraron dentro del rango de normalidad $\left(\mathrm{PtiO}_{2}>15 \mathrm{mmHg}\right.$ ) en más del $80 \%$ de los casos en que el lactato o el índice LP estuvieron elevados.

Conclusiones. El lactato e índice LP elevados fue un hallazgo frecuente después de un TCE y no se relacionó, en la mayoría de casos, con episodios de hipoxia tisular. Además, la concordancia entre los dos biomarcadores para clasificar una alteración metabólica fue débil. El índice LP y el lactato no deberían utilizarse indistintamente en la práctica clínica diaria debido a su débil correlación, su difícil interpretación y a su fisiopatología de naturaleza heterogénea y compleja. Cuando se detectan unos niveles de lactato o de índice LP elevados deberían ser consideradas otras causas a parte de la hipoxia tisular.

PALABRAS CLAVE: Hipoxia cerebral Índice lactatopiruvato. Lactato. Metabolismo anaeróbico. Microdiálisis. Traumatismo craneoencefálico

Is lactate a good indicator of brain tissue hypoxia in the acute phase of traumatic brain injury? Results of a pilot study in 21 patients

\section{Summary}

Lactate and the lactate-pyruvate index (LPI) are two hypoxia markers widely used to detect brain tissue hypoxia in patients with acute traumatic brain injury. These two markers have a more complex behavior than expected as they can be abnormally high in circumstan-

Abreviaturas. ATP: adenosin trifosfato. FSC: flujo sanguíneo cerebral. HIC: hipertensión intracraneal. LDH: lactato deshidrogenasa. LP: indice lactato/piruvato. MD: microdiálisis. $P D H$ : piruvato deshidrogenasa. $\mathrm{PtiO}_{2}$ : presión tisular de oxígeno. TC: tomografía computarizada. TCE: traumatismo craneoencefálico. 
ces with no detectable brain hypoxia. This condition must be considered in the differential diagnosis because it also reflects an alteration of brain energy metabolism.

Objectives. 1. To describe cerebral energy metabolism characteristics observed in the acute phase of traumatic brain injury (TBI) based on two traditional indicators of anaerobic metabolism: lactate and LPI, 2. To determine the concordance between these two biomarkers in order to classify the incidence of anaerobic metabolism and 3. To classify the different types of metabolic abnormalities found in patients with moderate and severe TBI using both lactate and LPI.

Materials and methods. Twenty-one patients were randomly selected from a cohort of moderate or severe TBI patients admitted to the neurotraumatology intensive care unit. All of them who underwent both cerebral microdialysis and brain tissue oxygen monitoring $\left(\mathrm{PtiO}_{2}\right)$. We analyzed the levels of lactate and the LPI for every microvial within the first 96 hours after head trauma. These data were correlated with $\mathrm{PtiO}_{2}$ values.

Results. Lactate levels and the LPI were respectively increased during $49,5 \%$ and $38,4 \%$ of the monitoring time. The incidence and behavior of high levels of both markers were extremely heterogeneous. The concordance between these two biomarkers to determine episodes of dysfucntional metabolism was very weak (Kappa Index=0,29; IC 95\%: 0,24-0,34). Based on the levels of lactate and the LPI, we defined four metabolic patterns: $\mathrm{I}: \mathrm{L}>\mathbf{2 , 5} \mathrm{mmol} / \mathrm{L}$ and $\mathrm{LPR}>25$; II: $\mathrm{L}>\mathbf{2 , 5}$ $\mathrm{mmol} / \mathrm{L}$ and $\mathrm{LPR} \leq 25$; $\mathrm{III}: \mathrm{L} \leq 2,5 \mathrm{mmol} / \mathrm{L}$ and $\mathrm{LPR} \leq$ 25; IV: $L \leq 2,5 \mathrm{mmol} / \mathrm{L}$ and $\mathrm{LPR}>25$ ). In more than $80 \%$ of cases in which lactate or LPI were increased, $\mathrm{PtiO}_{2}$ values were within the normal range $\left(\mathrm{PtiO}_{2}>\right.$ $15 \mathrm{mmHg})$.

Conclusions. Increased lactate and LPI were frequent findings after acute TBI and in most cases they were not related to episodes of brain tissue hypoxia. Furthermore, the concordance between both biomarkers to classify metabolic dysfunction was weak. LPI and lactate should not be used indistinctly in everyday clinical practice because of the weak correlation between these two markers, the difficulty in their interpretation and the heterogeneous and complex nature of the pathophysiology. Other differential diagnoses apart from tissue hypoxia should always be considered when high lactate and/or LPI are detected in the acute injured brain.

KEY WORDS: Brain hypoxia. Lactate. Lactate-pyruvate index. Microdialysis. Traumatic brain injury. Anaerobic metabolism.
Introducción

El encéfalo traumático es mucho más vulnerable que el encéfalo normal a los episodios de hipoxia tisular y a la hipertensión intracraneal (HIC) $)^{6,41}$. Esta especial sensibilidad se debe a las alteraciones casi constantes que la lesión cerebral traumática provoca en los mecanismos de regulación del flujo sanguíneo cerebral (FSC) ${ }^{28,29}$, en la permeabilidad de la barrera hematoencefálica ${ }^{29}$, y en la liberación masiva de aminoácidos excitatorios ${ }^{41}$. Estas alteraciones -denominadas lesiones secundarias, debido a que pueden aparecer minutos, horas o incluso días después del impacto inicial- pueden ser parcial o completamente reversibles y, por lo tanto, sensibles a diferentes maniobras terapéuticas.

La incidencia de episodios de hipoxia tisular cerebral en pacientes que han sufrido un TCE es elevada ${ }^{7}$. Por ello, y por su potencial reversibilidad, los sistemas de neuromonitorización, están en gran parte orientados a la prevención y diagnóstico precoz de la hipoxia cerebral. Entre las técnicas más recientemente introducidas en las unidades de pacientes neurocríticos destacan la monitorización de la presión tisular de oxígeno $\left(\mathrm{PtiO}_{2}\right)^{24} \mathrm{y}$ MD cerebral ${ }^{4}$.

Mientras que la $\mathrm{PtiO}_{2}$ indica la cantidad de oxígeno disuelto, y por lo tanto, la disponibilidad de oxígeno en el tejido cerebral, la MD cerebral permite conocer directamente cómo las células responden y adaptan su metabolismo ante las alteraciones en la disponibilidad de este oxígeno. La MD permite además detectar y estudiar otras alteraciones en el estado energético celular que no cursan con hipoxia tisular.

Los parámetros más utilizados como indicadores de hipoxia tisular son el lactato y el índice lactato-piruvato (LP) $)^{11,12,21,30,32}$. Estos dos indicadores se han utilizado indistintamente con el mismo objetivo debido a su elevada sensibilidad para detectar episodios de hipoxia tisular (Figura 1). Sin embargo, la interpretación de estos indicadores metabólicos no es sencilla ni se adapta en muchos casos a los conceptos fisiopatológicos tradicionales aceptados en la isquemia. Esto es en parte debido a que, el lactato y el índice LP, presentan características diferentes que deben tenerse en cuenta en su interpretación. Desde hace años se conoce que a nivel sistémico, existen pacientes críticos con aumentos significativos del lactato en los que no se detecta un insuficiente aporte de oxígeno. En el encéfalo, el lactato puede aumentar en ausencia de hipoxia tisular y esta circunstancia debe considerarse en el diagnóstico diferencial, puesto que traduce también una alteración, a menudo multifactorial, y siempre compleja en el metabolismo energético. Entre las posibles causas de aumento del lactato cerebral en condiciones de suficiente aporte de oxígeno se encuentran: 1) la disfunción de la enzima piruvato-deshidrogenasa $(\mathrm{PDH})$, como se ha demostrado en pacientes con sepsis ${ }^{9,26}$, 


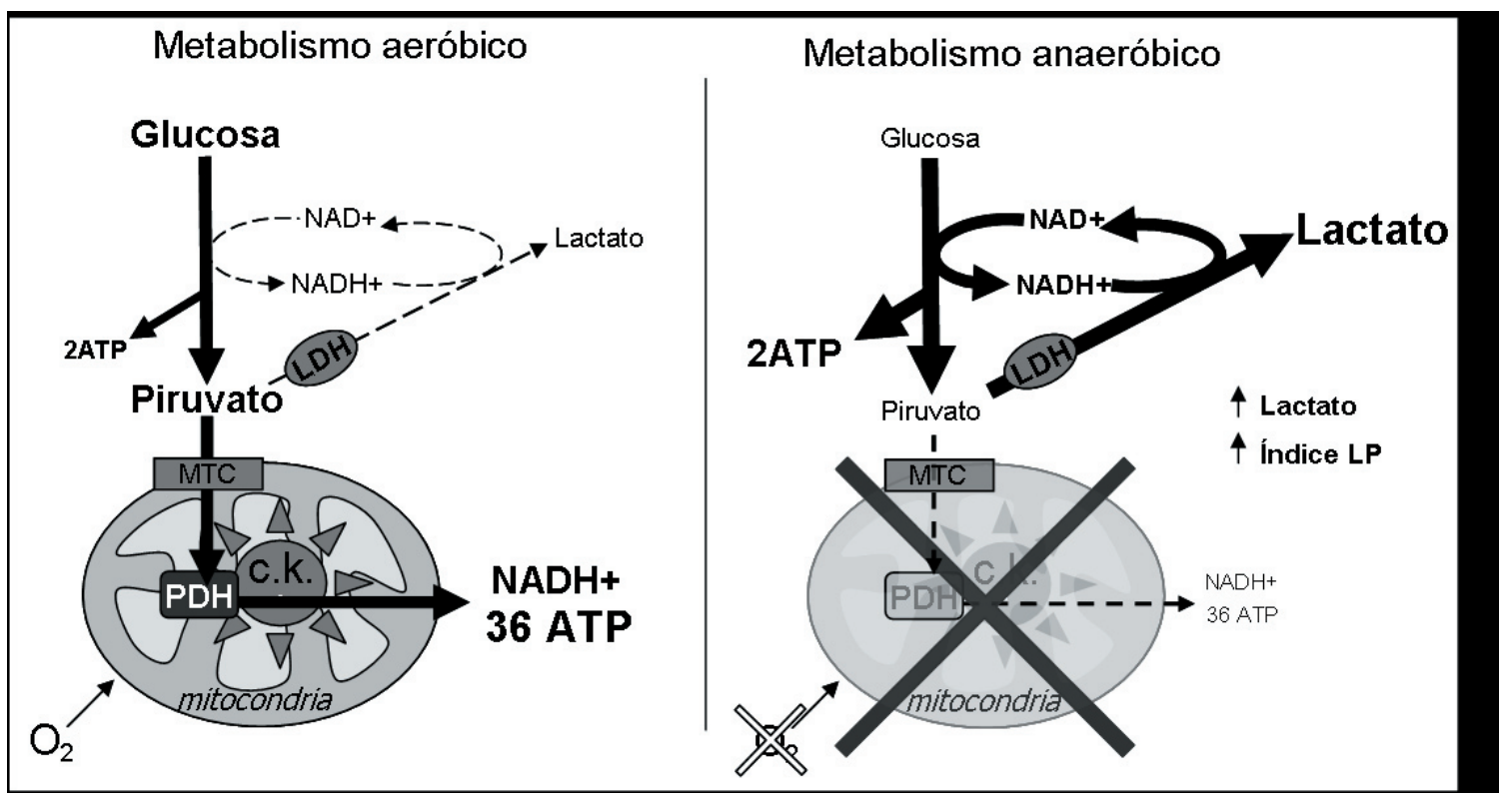

Figura 1. La figura muestra la diferencia metabólica entre una situación aeróbica y anaeróbica. El lactato y el índice lactato/piruvato se incrementan debido, por una parte a que el metabolismo aeróbico se detiene ante la falta de oxígeno y, por otra parte a que la velocidad de la glucólisis se incrementa para poder suplir con el máximo ATP posible la energía necesaria para la supervivencia de la célula. El nivel de producción de lactato y de funcionalidad de la mitocondria puede presentar estados intermedios entre la situación aeróbica y la anaeróbica dependiendo del tipo de alteración metabólica. ATP:adenosin trifosfato; LDH:enzima lactato deshidrogenasa; MTC:transportador para monocarboxilatos; $\mathrm{NADH}^{+}$:nicotinamida adenin nucleótido (forma reducida); $\mathrm{NAD}^{+}$:nicotinamida adenín nucleótido (forma oxidada); $\mathrm{O}_{2}:$ oxígeno PDH: enzima piruvato deshidrogenasa.

2) la disfunción mitocondrial ${ }^{38,42}, 3$ ) el hipermetabolismo cerebral con incrementos bruscos de la demanda energética estimulada por una liberación de glutamato $\left.{ }^{2,3}, 4\right)$ el incremento del amonio como ocurre en pacientes con disfunción hepática ${ }^{16}$ y 5) la hiperlactacidemia ${ }^{5,14,36}$.

De una forma tradicional se ha considerado que el índice LP es un indicador más específico que el lactato aislado para reflejar las alteraciones metabólicas inducidas por la hipoxia tisular ya que permite descartar como metabolismo anaeróbico algunos procesos hipermetabólicos en los que los niveles de lactato se elevan de forma importante ${ }^{37}$. Además este índice no se altera por las fluctuaciones en la recuperación del catéter de microdiálisis que con frecuencia ocurren en la práctica clínica diaria ${ }^{37}$. Recientemente, diferentes grupos de investigación han observado la existencia de incrementos del índice LP cerebral que, a pesar de ser significativos, no coincidían con ningún episodio de hipoxia tisular ${ }^{15,39}$. Al contrario, también se han descrito valores normales del índice LP en situaciones de hipoxia tisular profunda que cursan con incrementos transitorios tanto del piruvato como del lactato ${ }^{15}$.

Estas situaciones, pueden llevar a interpretaciones erróneas de la situación metabólica del paciente si no se tiene en cuenta el comportamiento del lactato y del piruvato por separado. Para ello es necesario profundizar en los meca- nismos por los cuáles las células obtienen energía para su supervivencia y función.

El piruvato es el metabolito que vincula la glucólisis con el metabolismo oxidativo o respiración celular que se produce de forma exclusiva en las mitocondrias ${ }^{10}$. Este vínculo depende de la competición entre dos enzimas capaces de metabolizar el piruvato: la lactato deshidrogenasa (LDH), que convierte el piruvato en lactato en el citosol, y la piruvato deshidrogenasa (PDH) que desvía el piruvato hacia su oxidación completa en condiciones aeróbicas en el ciclo de Krebs ${ }^{22}$ (Figura 1). La concentración tisular de piruvato depende, entre otros factores, de la velocidad de su síntesis por la glucólisis, de la velocidad de su utilización por la LDH y la PDH, y de la afinidad de estas dos enzimas por el piruvato.

En este estudio piloto pretendemos: 1) describir el comportamiento del metabolismo energético cerebral que se observa en la fase aguda de los pacientes que han sufrido un TCE en base a los dos indicadores tradicionales del metabolismo anaeróbico: el lactato y el índice LP, 2) determinar el nivel de concordancia de ambos indicadores para clasificar los episodios detectados de disfunción metabólica, y 3 ) estudiar el valor del lactato y del piruvato para clasificar los distintos tipos de alteraciones metabólicas que se encuentran en los pacientes con un TCE moderado o grave. 


\section{Material clínico y métodos}

En este estudio se incluyeron veintiún pacientes seleccionados de una forma aleatoria de una base de datos de 147 pacientes con criterios de TCE moderado o grave ingresados en la UCI de Neurotraumatología del Hospital Universitario Vall d'Hebrón y monitorizados con microdiálisis (MD) cerebral entre septiembre de 1999 y enero de 2008. Para la selección inicial se siguieron los siguientes criterios de inclusión: 1) TCE moderado o grave, 2) puntuación en la escala de Glasgow inferior o igual a 13, 3) edad entre 18 y 70 años, 4) TC cerebral en el momento de la implantación del catéter de microdiálisis cerebral con criterios de lesión difusa tipo II, III, IV ó V de acuerdo con la clasificación de Marshall et $\mathrm{al}^{20}$. Una vez implantado el catéter de MD cerebral no se descartaron aquellos pacientes con TCs que evolucionaran hacia una clasificación de tipo VI. 5) que tuvieran monitorización simultánea con un sensor de oximetría tisular, 6) que el sensor de MD estuviera implantado en un área macroscópicamente sana o alejada de lesiones hiperdensas por criterios de TC (Figura 2).

A cada paciente ingresado se le asignó de forma correlativa, por orden de ingreso, un número identificativo. Una persona ajena al estudio fue escogiendo de forma ciega y al azar entre los 147 números. Por ese orden se fueron incluyendo a los pacientes hasta que se obtuvieron 21 pacientes válidos, descartando todos aquellos pacientes que presentaron una monitorización mediante MD cerebral cuyos resultados fueran poco fiables: falta de recuperación de muestra del catéter de MD cerebral, fallo sistemático de algún reactivo, o cualquier problema técnico que no garantizara la fiabilidad de los resultados obtenidos. Por todos estos motivos se descartó a sólo un paciente.

Todos los pacientes fueron tratados según las guías de práctica clínica de la Brain Trauma Foundation enfocadas a la prevención de las lesiones secundarias, al tratamiento escalonado de HIC y a evitar la hipoxia cerebral.

Veinte de los pacientes incluidos se monitorizaron con un catéter de MD cerebral y uno de los pacientes con dos catéteres (CMA70 ó CMA71 high cut-off, CMA Microdialysis, Solna, Suecia). A este último paciente se le colocó un catéter en la zona de penumbra y otro en una zona sana del hemisferio contralateral a la lesión obteniendo de esta forma un total de veintidós zonas de parénquima cerebral monitorizadas mediante MD cerebral $(\mathrm{N}=22)$. No se ha descartado ninguno de estos dos catéteres aunque sean del mismo paciente puesto que son dos zonas de tejido cerebral macroscópicamente diferenciadas y ninguno de los catéteres sufrió problemas con la recuperación de microdializado. En todos los casos se implantó además un sensor intraparenquimatoso o intraventricular para monitorizar la PIC (Camino ${ }^{\mathrm{TM}}$, Integra Neurocare, USA),

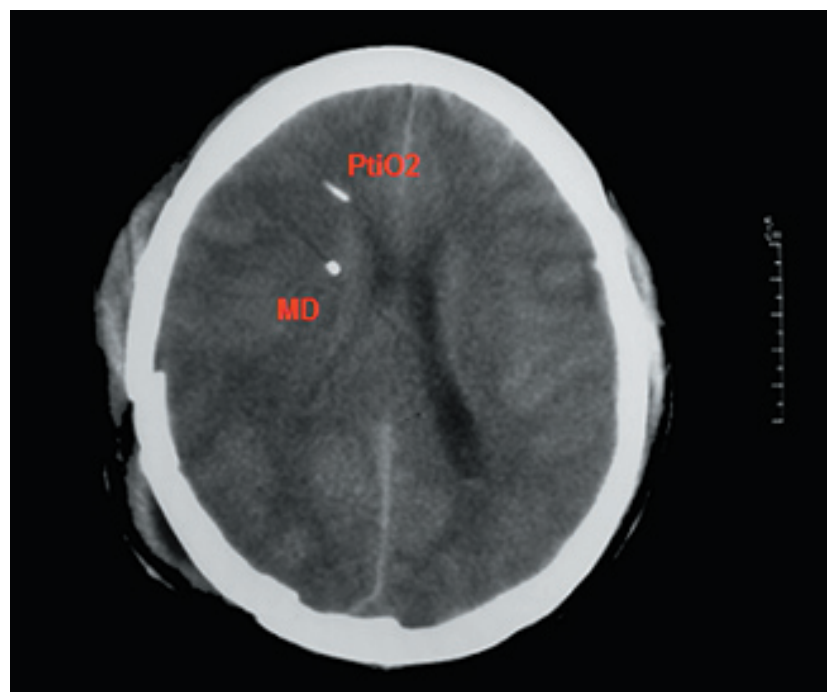

Figura 2 . TC craneal de un paciente al que se le implantó un catéter de microdiálisis cerebral (MD) y un catéter de oximetría tisular $\left(\mathrm{PtiO}_{2}\right)$.

y un sensor de oximetría tisular $\left(\mathrm{PtiO}_{2}\right)\left(\mathrm{Licox}^{\mathrm{TM}}\right.$, Integra Neurocare, USA). De los 21 pacientes incluidos en este estudio, a 16 se les colocó un catéter de MD cerebral convencional (cutoff: $20 \mathrm{KDa}$ ) y a 5 pacientes catéteres de MD cerebral de alta resolución (cutoff: $100 \mathrm{KDa}$ ). Hasta el año 2005 no aparecieron en el mercado los catéteres de MD de alta resolución. Esto permitió incrementar el rango de moléculas recuperables del espacio intersticial que podían ser estudiadas por lo que se decidió a partir de ese momento utilizar solamente este tipo de catéteres. La recuperación de estos analitos no queda alterada con el cambio de catéter ${ }^{1}$. La correcta localización del catéter fue comprobada siempre visualizando la punta del sensor mediante un TC de control.

De los veintidós catéteres de MD cerebral implantados en 21 pacientes, diecisiete se encontraban en el mismo hemisferio que el sensor de $\mathrm{PtiO}_{2}$. Los cinco catéteres restantes de microdiálisis se encontraban en el hemisferio contralateral al sensor de $\mathrm{PtiO}_{2}$. En estos cinco pacientes el tipo de lesión (difusa) permite asumir que los valores del hemisferio contralateral son representativos de la oxigenación cerebral global ${ }^{23}$. Para la perfusión de los catéteres de MD se utilizó un líquido de perfusión comercial (Líquido de perfusión CNS, CMA Microdialysis, Solna, Suecia) con la siguiente composición: $\mathrm{NaCl}(147 \mathrm{mM}), \mathrm{KCl}(2.7 \mathrm{mM})$, $\mathrm{CaCl}_{2}(1.2 \mathrm{mM}), \mathrm{MgCl}_{2}(0.85 \mathrm{mM})$. Los microviales se recogieron cada hora y se analizaron a la cabecera del paciente con el analizador CMA600 (Microdialysis Analyser, CMA Microdialysis, Solna, Suecia), para determinar los niveles de glucosa, lactato y piruvato. Para este estudio se tuvieron en cuenta sólo los microviales recogidos durante las primeras 96 horas tras el traumatismo. 


\section{Metodología utilizada para detectar errores y artefactos}

Los resultados obtenidos fueron procesados e integrados con las demás variables sistémicas del paciente mediante el programa LabPilot ${ }^{\mathrm{TM}}$ (versión 1.3, CMA Microdialysis, Solna Suecia). Estos datos fueron validados y depurados siempre por el mismo observador (MAM). De forma sistemática se desecharon los microviales recogidos durante las dos primeras horas post-implantación para evitar las fluctuaciones metabólicas propias del periodo de equilibrio que se produce después de la inserción del catéter de MD. En todos los casos se aseguró que todos los valores obtenidos fueran reales, descartando fallos técnicos como el intercambio accidental de microviales o resultados enmascarados por la falta de muestra en el microvial.

\section{Análisis estadístico}

Los datos de los diferentes metabolitos y variables del paciente se recogieron con el programa ICU Pilot 1.2 (CMA, Estocolmo, Suecia). Posteriormente, estos datos se exportaron para su análisis al programa SPSS versión 16.0 (SPSS Inc, Chicago, USA), Sigmastat 3.5 y SigmaPlot 9.0 (Systat Software Inc., California, USA). Debido a que nuestro estudio es fundamentalmente descriptivo se efectuó un análisis estadístico enfocado a este tipo de estudios. Se evaluó la distribución normal o no-normal de las variables cuantitativas mediante el método de Shapiro-Wilks. Las variables normales se describieron mediante la media y la desviación estándar y las variables que no siguieron una distribución normal se describieron con la mediana y los valores mínimo y máximo. Para determinar la asociación entre variables cuantitativas se utilizó el test de Pearson o de Spearman según la distribución de las variables. Las variables cualitativas se representaron mediante tablas de frecuencias con las frecuencias relativas y absolutas. Para comparar y medir la posible asociación entre las variables cualitativas se utilizó la prueba de homogeneidad de distribuciones discretas (Test Ji-Cuadrado). Para determinar el acuerdo entre los dos métodos de determinación del metabolismo anaeróbico (niveles de lactato e índice LP) se utilizó el índice kappa que calcula el acuerdo entre varios observadores corregido por el azar. El grado de significación estadística se estableció para un valor de $\mathrm{P}$ inferior o igual a 0,05 .

\section{Resultados}

Los pacientes incluidos ( 15 hombres y 6 mujeres) tenían una edad media de 34 años (Tabla 1). La clasificación de Marshall del TC de estos pacientes se muestra en la Tabla 1, así como la zona del tejido cerebral monitorizada para cada uno de los pacientes y los niveles de intensidad terapéutica a los que fueron sometidos para tratar la hipertensión intracraneal.
Tabla 1

Datos demográficos

\begin{tabular}{ccc} 
Sexo & Hombres & \\
& Mujeres & $15(73 \%)$ \\
Edad & $6(27 \%)$ \\
\hline GCS inicial & $34( \pm 13)$ \\
\hline Clasificación del TAC inicial & $6[$ rango: $4-10]$ \\
II & \\
III & $11(52 \%)$ \\
IV & $3(14 \%)$ \\
V & $1(5 \%)$ \\
VI & $1(5 \%)$ \\
Clasificación del TAC (evolución) & $5(24 \%)$ \\
II & $10(48 \%)$ \\
III & $4(19 \%)$ \\
IV & $0(0 \%)$ \\
V & $1(5 \%)$ \\
VI & $6(28 \%)$ \\
\hline
\end{tabular}

\begin{tabular}{l} 
Localización de los 22 catéteres \\
$\begin{array}{l}\text { de MD cerebral implantados } \\
\text { Zona no lesionada }\end{array}$ \\
$\quad \begin{array}{l}\text { Perilesional } \\
\text { Evolución de la zona monitorizada }\end{array}$ \\
\hline
\end{tabular}

Terapias de segundo nivel

$\begin{array}{lc}\text { Ninguna } & 14(64 \%) \\ \text { Barbitúricos } & 0(0 \%) \\ \text { Hipotermia } & 4(23 \%) \\ \text { Craniectomía } & 1(4 \%) \\ \text { Barbitúricos + Hipotermia } & 1(4 \%) \\ \text { Hipotermia + Craniectomia } & 1(4 \%)\end{array}$

GOSE 6 meses

6 [rango: 1-8]

* El primer caso de zona no lesionada a zona lesionada. El segundo de zona no lesionada a zona perilesional y el tercer caso de zona perilesional a zona no lesionada.

Episodios de incremento de lactato y del índice LP

Las medianas de los valores de lactato e índice LP que se encontraron en los 21 pacientes fue de 2,49 $\mathrm{mmol} / \mathrm{L}$ [min, max: 0,1-9,6] y de 22,9 [min, max: 8,26-163] respectivamente.

De los 21 pacientes se obtuvieron un total de 1.430 microviales que correspondían a 1.430 horas de monitorización. Un total de 708 microviales $(50,2 \%)$ presentaron unos niveles de lactato superiores a $2,5 \mathrm{mmol} / \mathrm{L}$ distribuidos en un total de 75 episodios. La duración de estos 


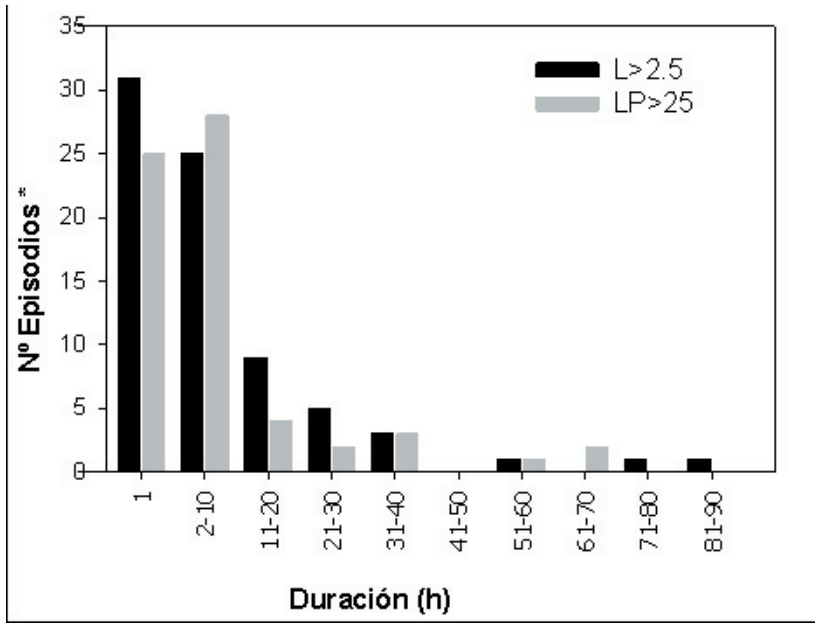

Figura 3. Representación de la duración de los episodios* de lactato e índice LP elevados $(2,5 \mathrm{mmol} / \mathrm{L}$ y 25 respectivamente). * Cada muestra analizada corresponde a una hora de monitorización. Como episodio se considera el número de muestras (horas) seguidas en las que el lactato y el indice LP se encuentran elevados.

episodios fue muy variable, siendo en 29 de ellos $(38,7 \%)$ episodios aislados de tan sólo una hora de duración. El resto de episodios osciló entre las 2 y las 82 horas según se muestra en la Figura 3.

Los valores del índice LP fueron superiores a 25 en 544 microviales $(38,7 \%)$, equivalente a 544 horas repartidas en 65 episodios. La duración de estos episodios, al igual que para el lactato, fue muy variable aunque con una mayor frecuencia de episodios inferiores a las 10 horas (Figura 3).

Tanto los episodios de incremento de lactato como del índice LP presentaron una incidencia y un comportamiento muy heterogéneos entre los diferentes pacientes. Por ejemplo, mientras que solamente tres pacientes no presentaron ningún valor de lactato por encima de $2,5 \mathrm{mmol} / \mathrm{L}$, cuatro pacientes presentaron valores persistentemente elevados durante el $100 \%$ del tiempo de monitorización. El resto de pacientes presentaron un número de episodios y un porcentaje de tiempo con valores de lactato extremadamente variables (Tabla 2).

\section{Concordancia entre métodos}

Se analizó el nivel de concordancia entre los dos indicadores como marcadores de metabolismo anaeróbico. Para el lactato, niveles superiores a $2,5 \mathrm{mmol} / \mathrm{L}$ se clasificaron como indicativos de metabolismo anaeróbico. Para el índice LP, se clasificó como metabolismo anaeróbico aquellos valores con índice LP superior a 25. Estos umbra-

TABLA 2

Distribución y frecuencia de los episodios de L e índice LP para cada paciente

\begin{tabular}{|c|c|c|c|c|c|}
\hline Paciente & $\begin{array}{l}\text { Tiempo }(\mathrm{h}) \\
\text { monitorización }\end{array}$ & $\begin{array}{l}\mathrm{n}^{\mathrm{o}} \text { Episodios } \\
\mathrm{L}>2,5 \mathrm{mM}\end{array}$ & $\begin{array}{c}\% \text { Tiempo } \\
\text { L }>2,5\end{array}$ & $\begin{array}{l}\mathrm{n}^{\circ} \text { Episodios } \\
\text { índice } \mathrm{LP}>25\end{array}$ & $\begin{array}{l}\% \text { Tiempo } \\
\text { índice LP }>25\end{array}$ \\
\hline 1 & 64 & 0 & 0,0 & 2 & 5,3 \\
\hline 2 & 85 & 9 & 21,2 & 6 & 34,1 \\
\hline 3 & 70 & 9 & 44,3 & 4 & 75,7 \\
\hline 4 & 85 & 8 & 61,2 & 3 & 96,2 \\
\hline 5 & 40 & 2 & 70,0 & 2 & 27,5 \\
\hline 6 & 52 & 3 & 94,2 & 2 & 53,8 \\
\hline $7 \mathrm{D}$ & 35 & 1 & 100 & 1 & 100 \\
\hline $7 \mathrm{I}$ & 35 & 1 & 100 & 4 & 21,9 \\
\hline 8 & 78 & 1 & 100 & 5 & 50,0 \\
\hline 9 & 82 & 1 & 100 & 1 & 81,8 \\
\hline 10 & 72 & 7 & 73,6 & 6 & 20,8 \\
\hline 11 & 79 & 0 & 0,0 & 5 & 19,0 \\
\hline 12 & 66 & 4 & 43,9 & 2 & 31,8 \\
\hline 13 & 85 & 8 & 67,1 & 0 & 0,0 \\
\hline 14 & 27 & 1 & 88,9 & 1 & 88,9 \\
\hline 15 & 50 & 1 & 2,0 & 0 & 0,0 \\
\hline 16 & 54 & 1 & 100 & 4 & 9,4 \\
\hline 17 & 68 & 6 & 89,7 & 2 & 79,4 \\
\hline 18 & 65 & 0 & 0,0 & 0 & 0,0 \\
\hline 19 & 80 & 2 & 2,5 & 8 & 21,3 \\
\hline 20 & 81 & 1 & 17,3 & 0 & 0,0 \\
\hline 21 & 88 & 9 & 15,9 & 7 & 63,6 \\
\hline
\end{tabular}


Tabla 3

Clasificación y concordancia de cada una de las muestras analizadas del total de pacientes en base a los niveles de lactato e índice LP

\begin{tabular}{|c|c|c|c|c|}
\hline \multicolumn{5}{|c|}{ ÍNDICE LP } \\
\hline & & $\begin{array}{l}\text { Metabolismo anaeróbico } \\
\text { Índice LP }>25\end{array}$ & $\begin{array}{l}\text { Metabolismo no anaeróbico } \\
\text { Índice LP } \leq 25\end{array}$ & Total \\
\hline \multirow{3}{*}{ LACTATO } & $\begin{array}{l}\text { Metabolismo anaeróbico } \\
\mathrm{L}>2,5 \mathrm{mmol} / \mathrm{L}\end{array}$ & $\begin{array}{c}377 \\
(26.8 \%)\end{array}$ & $\begin{array}{c}331 \\
(23.4 \%)\end{array}$ & 708 \\
\hline & $\begin{array}{l}\text { Metabolismo no anaeróbico } \\
\mathrm{L} \leq 2,5 \mathrm{mmol} / \mathrm{L}\end{array}$ & $\begin{array}{c}167 \\
(11.9 \%)\end{array}$ & $\begin{array}{c}534 \\
(37.9 \%)\end{array}$ & 701 \\
\hline & Total & 544 & 865 & 1409 \\
\hline
\end{tabular}

les se seleccionaron en base a los más aceptados en la literatura como indicativos de un metabolismo anaerobio incrementado.

La concordancia entre los dos indicadores fue de sólo un 64,7\% (Tabla 3). El índice Kappa mostró un nivel de concordancia débil entre ambos indicadores metabólicos ( $\mathrm{K}=0,29$; IC 95\%: 0,24-0,34).

\section{Patrones metabólicos}

En base a los valores de lactato y del índice LP se definieron cuatro patrones metabólicos:

Patrón 1: lactato e índice LP elevados $(\mathrm{L}>2,5 \mathrm{mmol} / \mathrm{L}$ y $\mathrm{LP}>25)$

Patrón 2: lactato elevado e índice LP normal ( $\mathrm{L}>2,5$ $\mathrm{mmol} / \mathrm{L}$ y $\mathrm{LP} \leq 25)$

Patrón 3: lactato normal e índice LP elevado $(\mathrm{L} \leq 2,5$ $\mathrm{mmol} / \mathrm{L}$ y $\mathrm{LP}>25$ )

Patrón 4: lactato e índice LP normales $(\mathrm{L} \leq 2,5 \mathrm{mmol} / \mathrm{L}$ y $\mathrm{LP} \leq 25)$

La incidencia de estos cuatro patrones en la serie total de microviales analizados en los 21 pacientes se muestra en la Tabla 3. El patrón mas frecuente fue el Patrón 4 (534 microviales, 38\%). Este patrón indica en principio un metabolismo energético normal. En el $62 \%$ de microviales restantes, los niveles de lactato, el índice LP o ambos se encontraron elevados ( 875 microviales). El segundo patrón en frecuencia fue el Patrón 1, correspondiente al patrón clásico de hipoxia tisular de tipo isquémico o no isquémico.

\section{Alteraciones energéticas y $\mathrm{PtiO}_{2}$}

Para cada microvial se determinó el valor medio de $\mathrm{PtiO}_{2}$ correspondiente a cada hora analizada. Se observó que la mayor parte de los microviales que presentaban un lactato o un índice LP elevados no se asociaban a valores de $\mathrm{PtiO}_{2}$ indicativos de hipoxia tisular $\left(\mathrm{PtiO}_{2}<15 \mathrm{mmHg}\right)$ (Figura 4). El incremento de uno o de los dos marcadores de metabolismo "anaerobio" se dio en presencia de niveles de oxígeno superiores a $15 \mathrm{mmHg}$ en más del $80 \%$ de los casos.

\section{Discusión}

El lactato y el índice LP han sido las dos variables más utilizadas en la literatura como indicadores de metabolismo anaeróbico en cualquier órgano. Las nuevas técnicas de MD permiten valorar estos índices en el parénquima encefálico del paciente crítico aunque aportan sin embargo datos contradictorios y de difícil interpretación. Esto coincide con una revisión de los fundamentos de la fisiopatología del transporte de oxígeno y del metabolismo anaeróbico tanto a nivel cerebral como sistémico que cuestiona muchos de los postulados considerados tradicionalmente consolidados. A nivel del encéfalo, y en especial del encéfalo lesionado, existen también numerosas paradojas, difíciles de explicar que las técnicas de microdiálisis están poniendo de manifiesto y cuya resolución ayudarán sin duda a conocer mejor el metabolismo energético normal, sus alteraciones y su potencial modificación con determinadas maniobras terapéuticas.

\section{Niveles de lactato e índice $L P$}

Los valores medios que se obtuvieron de lactato y de índice LP en la serie completa de microviales resultan difícil de interpretar por la carencia de valores de normalidad de estos parámetros en individuos sanos. Reinstrup et al. ${ }^{25}$ (Tabla 4) ofrecen una aproximación de los niveles basales de estos marcadores que han sido determinados monitorizando una zona sana de tejido cerebral en pacientes 

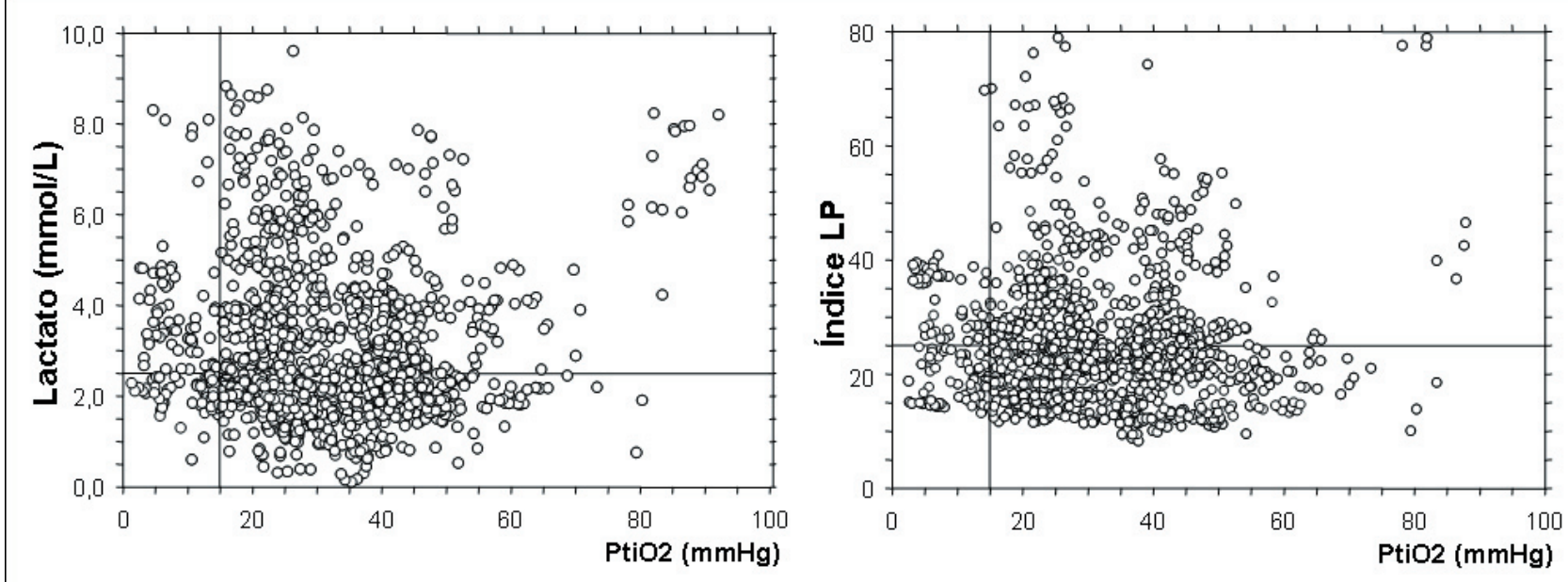

Figura 4. Valores de PtiO, vs. lactato e índice LP. La mayor parte de las determinaciones de lactato y de índice LP por encima de 2,5mmol/L y 25 respectivamente, se asocian a valores de $P t i O$ por encima de $15 \mathrm{mmHg}$. En el caso del indice LP se observa incluso cómo a partir de 40 no hay prácticamente lecturas de $\mathrm{PtiO}_{2}$ por debajo de $15 \mathrm{mmHg}$.

no traumáticos sometidos a cirugía de tumores de la fosa posterior. Para el lactato, Reinstrup et al. ${ }^{25}$ observaron unos valores superiores en los pacientes despiertos que el subgrupo de pacientes que se encontraban sedados. La mayor actividad cerebral que presentan los individuos despiertos justifica estos hallazgos al tener éstos una mayor demanda energética y un mayor consumo de oxígeno y glucosa ${ }^{17}$. Según la reciente hipótesis de la lanzadera de lactato entre neuronas y astrocitos postulada por Pellerin y Magisttretti ${ }^{18}$, durante la actividad cerebral, la producción de lactato en los astrocitos se incrementa estimulada por el glutamato liberado por las neuronas en la hendidura sináptica, este lactato es excretado por los astrocitos y captado por las neuronas como fuente de energía para suplir la demanda energética puntual que se requiere ante la activación neuronal.
Los niveles de lactato en nuestra serie se encontraron elevados respecto a los niveles de lactato de la serie de pacientes no traumáticos y sedados de Reinstrup et al $(1,20 \mathrm{mmol} / \mathrm{L})$. Es conocido que la demanda energética en los pacientes con lesiones cerebrales agudas es muy superior a la de los individuos normales. A pesar de ello los niveles de $\mathrm{PtiO}_{2}$ medidos en la proximidad del catéter de MD fueron normales en más del $85 \%$ de las lecturas en las que el lactato se encontró elevado (Figura 4). Este hallazgo confirmaría que las causas de elevación de lactato son en la mayoría de nuestros pacientes de etiología no hipóxica.

Por otra parte, si comparamos estos resultados con el estudio de Shultz et al..$^{31}$, en donde estos autores determinaron los niveles de lactato e índice LP durante episodios de isquemia en pacientes con hemorragia subaracnoidea (Tabla 4), observamos que éstos fueron mucho más

TABLA 4

Valores "normales" y umbrales de isquemia del lactato e índice LP

\begin{tabular}{|c|c|c|c|c|}
\hline Marcador & $\begin{array}{l}\text { Serie de pacientes de este esudio } \\
\text { (mediana [IQ]) }\end{array}$ & \multicolumn{2}{|c|}{$\begin{array}{l}\text { Reinstrup et al. } .^{25} \\
\text { (media + DS) }\end{array}$} & $\begin{array}{l}\text { Schulz et al. }{ }^{31} \\
\text { (media + DS) }\end{array}$ \\
\hline Grupos & Valores TCE & Sedados & Despiertos & Grupo isquémico \\
\hline Lactato (mmol/L) & $2,49[0,105-9,6]]$ & $1,20 \pm 0,60$ & $2,90 \pm 0,90$ & $6,73 \pm 1,09$ \\
\hline Índice LP & $22,9[8,26-163]$ & $22,0 \pm 6,00$ & $23,0 \pm 4,00$ & $97,8 \pm 32,2$ \\
\hline Características & TCE & \multicolumn{2}{|c|}{ Tumores fosa posterior } & HSA de aneurisma \\
\hline
\end{tabular}

SD.: desviación estandar; IQ: rango intercuartil [IQ 25\%-IQ 75\%] 
elevados que en nuestra serie de pacientes en la que se estudió un perfil metabólico más heterogéneo. El hecho de que nuestros valores de lactato sean más elevados que en el grupo de pacientes no traumáticos, pero mucho más bajos que el grupo en el que solamente se estudia el patrón isquémico sugiere que existen alteraciones más moderadas en los niveles de lactato que no cursan con hipoxia pero que pueden ser características en el contexto de un TCE.

En nuestra serie, el índice LP, no se encontró muy elevado respecto a los valores de normalidad publicados por Reinstrup et al. (Tabla 4). Esto concuerda con la idea de que el comportamiento del índice LP no es necesariamente representativo del comportamiento del lactato aislado como se demuestra en las Tablas 2 y 3 . El nivel de concordancia entre estos dos indicadores metabólicos fue bajo. Además, la mayor parte del tiempo en que el índice LP se encontró elevado, los valores de $\mathrm{PtiO}_{2}$ también fueron normales (Figura 4) lo que sugiere que este indicador tampoco es muy específico a la hora de determinar episodios de hipoxia tisular. Esto podría sugerir una elevada prevalencia de disfunción mitocondrial tras un TCE grave (hipoxia histotóxica de la clasificación de Siggäard-Andersen) o una alteración de las enzimas clave que regulan la glucólisis y el paso del piruvato al ciclo de Krebs. Xiaog et al. ${ }^{42}$ ya demostraron la existencia de disfunción mitocondrial en modelos animales de TCE. Posteriormente Verweij et al. ${ }^{38}$ lo demostraron directamente en un estudio realizado con una serie de 16 pacientes que habían sufrido un TCE. Esta disfunción mitocondrial se caracterizaba por una alteración en la producción de ATP y por un incremento del calcio intramitocondrial. La disminución en la producción de ATP por parte de la mitocondria produciría un aumento en la velocidad de la glucólisis que se traduciría en un incremento del índice LP. Esta disfunción mitocondrial daría lugar a un segundo tipo de hipoxia que puede cursar en presencia de unos niveles de oxígeno normales y que se denomina hipoxia por desacoplamiento según la clasificación de Siggäard-Andersen ${ }^{33}$.

\section{Prevalencia del lactato e índice LP elevado y su compor- tamiento temporal}

A pesar de que los niveles medios de lactato e índice LP de nuestra serie de pacientes no resultaran ser elevados respecto al grupo isquémico de Shultz, la incidencia y prevalencia de estos marcadores elevados en el perfil temporal de estos pacientes fue muy elevada (49,5\% para el lactato y 38,7\% para el índice LP) (Tabla 3). Además el 90\% de los pacientes presentó al menos un episodio con lactato cerebral superior a $2,5 \mathrm{mmol} / \mathrm{L}$ y un $80 \%$ de los pacientes lo presentó con un índice LP por encima de 25 (Tabla 2). Más de la mitad (57\%) estuvo durante más del 50\% del tiempo con unos niveles de lactato por encima de $2,5 \mathrm{mmol} / \mathrm{L}$. Esta alta frecuencia de episodios con elevación del lactato y del índice LP ha sido previamente descrita en estudios sobre

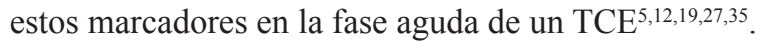

Un aspecto observado en nuestro estudio es que aproximadamente el $40 \%$ de los episodios de elevación del lactato y del índice LP aparecieron de forma aislada y tuvieron una duración relativamente corta (una o dos horas de duración) (Figura 3). Esto debe tenerse en cuenta puesto que sugiere un comportamiento altamente inestable pero reversible en el estado metabólico del encéfalo tras un TCE grave. Esta inestabilidad y heterogeneidad en el perfil temporal del metabolismo energético de estos pacientes podría ser más determinante en la fisiopatología del TCE que sus niveles en valores absolutos. Los cambios en la velocidad de producción o en el consumo de metabolitos como la glucosa, el lactato y el piruvato pueden dar una visión más real de la situación metabólica y fisiopatológica del tejido cerebral tras un TCE. Las enzimas implicadas en las reacciones son las generadoras y limitadoras de los niveles de estos metabolitos en el parénquima cerebral así como los mejores indicadores de la actividad celular.

\section{Índice $\mathbf{L P}$}

En un 38\% del tiempo total monitorizado se observó un índice LP elevado (patrón I y III) (Tabla 3). Como ya se ha indicado anteriormente, la mayoría de estos episodios coexistían con valores de $\mathrm{PtiO}_{2}$ por encima de $15 \mathrm{mmHg}$ (Figura 4). Vespa et al. ${ }^{40}$ ya detectaron en un estudio con pacientes que habían sufrido un TCE grave, índices LP elevados que no se correspondían con signos de isquemia regional evaluados por tomografía de emisión de positrones y $\mathrm{PtiO}_{2}$. Este estudio sugería también que la incidencia de isquemia tras un TCEG es un fenómeno menos común de lo que se cree a pesar de la elevada incidencia de valores elevados del índice LP en estos pacientes.

En la mitad de los episodios de índice LP elevados, no se detectó un lactato elevado (patrón III) indicando por lo tanto una reducción en los niveles intersticiales de piruvato (Tabla 3). Htlaky et al. ${ }^{15}$, ya observaron esta situación. Aquí se postulaba que este patrón podría deberse a un aporte reducido de glucosa o a una disfunción en la vía glicolítica, frecuente en los TCE.

En el presente estudio este patrón fue el menos frecuente (Tabla 3). Algunos de estos episodios se caracterizaron por una disminución brusca y reversible en los niveles de piruvato que podría sugerir una intensa carboxilación del piruvato para sintetizar glutamato a través de la ruta anaplerótica del ciclo de $\mathrm{Krebs}^{13}$. En la Figura 5 se observa un ejemplo de este comportamiento observado en un paciente con TCE y monitorizado mediante microdiálisis cerebral. Ante una liberación masiva de glutamato por parte de las neuronas podría necesitarse una gran cantidad de piruvato para suplir la disminución de los niveles de glutamato después de su liberación. 


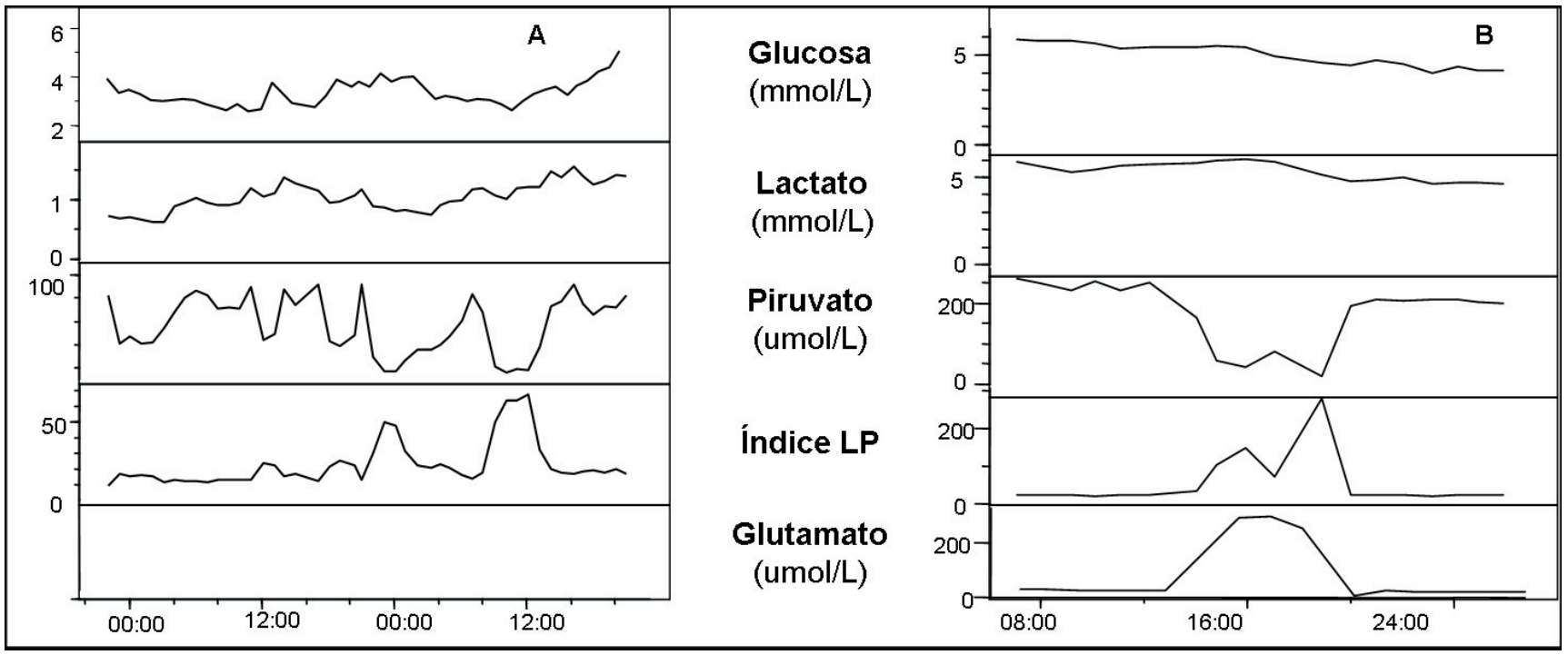

Figura 5. A. Se representa el patrón III caracterizado por unos niveles de lactato normales y unos índices LP elevados causados por depleciones de piruvato no explicadas por una depleción de glucosa. A pesar de valores de lactato bajos el indice LP se incrementa por encima de 50. Este comportamiento es reversible, los valores del índice LP tan elevados se recuperan en pocas horas. B. Se observa el mismo comportamiento dinámico a pesar de observarse unos niveles de lactato elevados. La depleción de piruvato coincide en este caso con un incremento súbito de glutamato y provoca igualmente un gran incremento del índice LP.

Vespa et al. ${ }^{39}$ propusieron además que un índice LP elevado, por sí solo no podía ser diagnóstico de episodios isquémicos y observaron que solamente un índice LP superior a 40 en combinación con niveles de glucosa cerebral inferiores a $0,4 \mathrm{mmol} / \mathrm{L}$ se correlacionaron con episodios de isquemia tisular determinados por tomografía de emisión de positrones. Estos hallazgos, al igual que nuestros resultados (Figura 4), sugieren que el índice LP es un indicador de hipoxia isquémica menos específico de lo que se había sugerido en estudios anteriores.

El estudio de Hlatky et al. ${ }^{15}$ mencionado anteriormente, demostró incrementos transitorios de piruvato y lactato que resultaban en una disminución del índice LP. Después el piruvato volvía a descender a valores cercanos a cero incrementándose de este modo el índice LP. Este patrón de comportamiento (también observado en nuestra serie de pacientes) lo encontraron en algunos pacientes coincidiendo con un episodio de isquemia profundo (determinado por unos niveles de $\mathrm{PtiO}_{2}$ muy bajos).

Estos episodios de isquemia con índices LP no elevados podrían tener una explicación desde el punto de vista bioquímico: ante una rápida depleción de oxígeno, y un incremento en la demanda energética, se produce un aumento de los niveles de piruvato a una velocidad mayor de la que la enzima LDH puede oxidarlo. Por otra parte la enzima PDH tampoco podría utilizar el piruvato puesto que la falta de oxígeno detendría el ciclo de Krebs debido a la acumulación de sustratos del ciclo de Krebs.

\section{Lactato}

Los estudios sobre hiperlactacidemia y acidosis láctica $^{34}$ son un buen ejemplo sobre las causas del incremento de los niveles de lactato arterial en ausencia de episodios de hipoperfusión que podrían darse también a nivel cerebral. Estas causas podrían también afectar a los niveles de lactato cerebral puesto que éste atraviesa la barrera hematoencefálica gracias a los transportadores de los monocarboxilatos. De hecho existen varios estudios que sugieren que un incremento de lactato arterial provoca un incremento de lactato cerebral ${ }^{5,14}$. Como hallazgos interesantes recientes, se ha demostrado que, en deportistas, los lactatos elevados en plasma pueden servir de sustrato aeróbico al sistema nervioso central ${ }^{8}$. En nuestra serie de pacientes, el $16.8 \%$ del tiempo en que las determinaciones de lactato cerebral estuvieron por encima de $2,5 \mathrm{mmol} / \mathrm{L}$ también presentaron unos niveles de lactato sistémico por encima de $3 \mathrm{mmol} / \mathrm{L}$ (datos no presentados). Hay que recordar que la glicólisis está regulada por un número elevado de enzimas que pueden alterar y desviar las rutas del metabolismo hacia otras vías sin necesidad de que la hipoxia tisular esté presente.

\section{Conclusiones}

La elevada prevalencia del incremento de lactato y del índice LP elevados no tuvo en su mayoría relación con episodios de hipoxia tisular. Los diferentes significados que 
pueden tener unos índices LP y lactato elevados hacen que estos dos indicadores no puedan ser utilizados indistintamente en la práctica clínica diaria y explicaría la débil concordancia entre estos dos marcadores como indicadores de metabolismo anaeróbico. El grado de especificidad de cada uno hace que, de momento, sea necesario el estudio conjunto con otros parámetros como son los niveles de glucosa y la presión tisular de oxígeno para poder discernir cuándo los niveles de L o índice LP están indicando una lesión de tipo hipóxica, ya sea isquémica o no.

Además, la elevada inestabilidad en los niveles de estos dos marcadores en el perfil temporal de las primeras 96 horas tras el accidente requiere una caracterización más precisa de los diferentes patrones y de los perfiles temporales observados para poder, en un futuro, ofrecer un diagnóstico diferencial del tipo de crisis metabólicas que se dan en este tipo de pacientes y su posible manipulación terapéutica.

\section{Agradecimientos}

Este estudio ha sido financiado parcialmente por el Fondo de Investigación Sanitaria de la Seguridad Social (beca FIS 07/0681 concedida al Dr. J. Sahuqui1lo) y por la Generalitat de Catalunya (beca predoctoral AGAUR -2008FI 00747. concedida a María A. Merino). Agradecemos la colaboración e inestimable ayuda de todo el equipo de enfermería de la Unidad de Cuidados Intensivos de Neurotraumatología del Hospital Universitario Vall d'Hebrón y de los Residentes del Servicio de Neurocirugía.

\section{Bibliografía}

1. Afinowi, R., Tisdall, M., Keir, G..et.al.: Improving the recovery of S100B protein in cerebral microdialysis: implications for multimodal monitoring in neurocritical care. J Neurosci Methods. 2009; 181: 95-99.

2. Alessandri, B., Doppenberg, E.M., Bullock, M.R., et.al.: Glucose and metabolism after severe human head injury: influence of exitatory neurotransmitters and injury type. Acta Neurochir Suppl (Wien ). 1999; 75: 21-24.

3. Alessandri,B., Doppenberg, E.M., Zauner, A., et al.: Evidence for time-dependent glutamate-mediated glycolysis in head-injured patients: a microdialysis study. Acta Neurochir Suppl (Wien ). 1999; 75: 25-28.

4. Bellander, B.M., Cantais, E., Enblad, P., et al.: Consensus meeting on microdialysis in neurointensive care. Intensive Care Med. 2004; 30: 2166-2169.

5. Chen, T., Qian, Y. Z., Di, X., Zhu, J. P., Bullock, R.: Evidence for lactate uptake after rat fluid percussion brain injury. Acta Neurochir Suppl 2000; 76: 359-364.

6. Coles, J.P.: Regional ischemia after head injury. Curr
Opin Crit Care. 2004; 10: 120-125.

7. Coles, J.P., Fryer, T.D., Smielewski, P., et al.: Incidence and mechanisms of cerebral ischemia in early clinical head injury. J Cereb Blood Flow Metab. 2004; 24: 202-211.

8. Dalsgaard, M.K.: Fuelling cerebral activity in exercising man. J Cereb Blood Flow Metab 2006 Jun. 2006; 26: 731750.

9. Fink, M.P.: Cytopathic hypoxia. Mitochondrial dysfunction as mechanism contributing to organ dysfunction in sepsis. Crit Care Clin. 2001; 1: 219-237.

10. Gjedde, A., Marrett, S.: Glycolysis in neurons, not astrocytes, delays oxidative metabolism of human visual cortex during sustained checkerboard stimulation in vivo. J Cereb Blood Flow Metab. 2001; 21: 1384-1392.

11. Goodman, J.C., Gopinath, S.P., Valadka, A.B., et al.: Lactic acid and amino acid fluctuations measured using microdialysis reflect physiological derangements in head injury. Acta Neurochir Suppl (Wien ). 1996; 67: 37-39.

12. Goodman, J.C., Valadka, A.B., Gopinath, S.P., Uzura, M., Robertson, C.S.: Extracellular lactate and glucose alterations in the brain after head injury measured by microdialysis. Crit Care Med. 1999; 27: 1965-1973.

13. Hassel, B.: Pyruvate carboxylation in neurons. J Neurosci Res. 2001; 66: 755-762.

14. Hellmann, J., Vannucci, R.C., Nardis, E.E.: Bloodbrain barrier permeability to lactic acid in the newborn dog: lactate as a cerebral metabolic fuel. Pediatr Res. 1982; 16: 40-44.

15. Hlatky, R., Valadka, A.B., Goodman, J.C., Contant, C.F., Robertson, C.S.: Patterns of energy substrates during ischemia measured in the brain by microdialysis. $\mathrm{J}$ Neurotrauma. 2004; 21: 894-906.

16. Kala, G., Hertz, L.: Ammonia effects on pyruvate/ lactate production in astrocytes--interaction with glutamate. Neurochem Int. 2005; 47: 4-12.

17. Magistretti, P.J.: Neuron-glia metabolic coupling and plasticity. J Exp Biol. 2006; 209: 2304-2311.

18. Magistretti, P.J., Pellerin, L.: Cellular mechanisms of brain energy metabolism and their relevance to functional brain imaging. Philos Trans R Soc Lond B Biol Sci. 1999; 354: 1155-1163.

19. Makoroff, K.L., Cecil, K.M., Care, M., Ball, W.S., Jr.: Elevated lactate as an early marker of brain injury in inflicted traumatic brain injury. Pediatr Radiol. 2005; 35: 668-676.

20. Marshall, L.F., Eisenberg, H.M., Jane, J.A., et al.: A new classification of head injury based on computerized tomography. J Neurosurg. 1991; 75: S14-S20.

21. Muller, M.: Science, medicine, and the future: Microdialysis. BMJ. 2002; 324: 588-591.

22. Nelson D.L., and Cox, M.M.: Lehninger Principles of Biochemistry. 2004.

23. Nortje, J., Gupta, A. K.: The role of tissue oxygen monitoring in patients with acute brain injury. Br J Anaesth. 2006; 97: 95-106. 
24. Poca, M.A., Sahuquillo, J., Mena, M.P., Vilalta, A., Riveiro, M.: [Recent advances in regional cerebral monitoring in the neurocritical patient: brain tissue oxygen pressure monitoring, cerebral microdialysis and near-infrared spectroscopy.]. Neurocirugía. 2005; 16: 385-410.

25. Reinstrup, P., Stahl, N., Mellergard, P., et al.: Intracerebral microdialysis in clinical practice: baseline values for chemical markers during wakefulness, anesthesia, and neurosurgery. Neurosurgery. 2000; 47: 701-200.

26. Ruza, F.: Tratado de cuidados intensivos pedriáticos. 2003.

27. Sahuquillo, J., Poca, M.A., Garnacho, A., et al.: Early ischaemia after severe head injury. Preliminary results in patients with diffuse brain injuries. Acta Neurochir (Wien ). 1993; 122: 204-214.

28. Sahuquillo, J., Rodriguez-Baeza, A., Baguena, M., et al.: Autorregulación cerebral: conceptos fisiopatológicos y metodología para su valoración en el paciente neurotraumatizado. Medicina intensiva. 1996; 20: 69-78.

29. Sahuquillo, J., Rodriguez-Baeza, A., Reina, F., Poca, M.A., Ferrer, A.M., Baguena, M.: Alteraciones en la reactividad al $\mathrm{CO} 2$ y la autorregulación en los traumatismos craneoencefálicos. Complicaciones terapéuticas, in Net A, Marruecos-Sant L (eds): Traumatismo craneoencefálico grave. Barcelona, Springer-Verlag Ibérica, 1996, pp 157-180.

30. Sarrafzadeh, A.S., Kiening, K.L., Callsen, T. A., Unterberg, A.W.: Metabolic changes during impending and manifest cerebral hypoxia in traumatic brain injury. Br J Neurosurg. 2003; 17: 340-346.

31. Schulz, M.K., Wang, L.P., Tange, M., Bjerre, P.: Cerebral microdialysis monitoring: determination of normal and ischemic cerebral metabolisms in patients with aneurysmal subarachnoid hemorrhage. J Neurosurg. 2000; 93: 808-814.

32. Siddiqui, M.M., Shuaib, A.: Intracerebral microdialysis and its clinical application: a review. Methods. 2001; 23: 83-94.

33. Siggaard-Andersen, M., Ulrich, A., Gothgen, I.H.: Classes of tissue hypoxia. Acta Anaesthesiol Scand. 1995; 39 : 137-142.

34. Stacpoole, P.W.: Lactic acidosis. Endocrinology and metabolism clinics of north america. 1993; 22: 221-245.

\section{Comentario al trabajo: ¿Es el lactato un buen indicador de hipoxia tisular en la fase aguda de la lesión cerebral traumática? Resultados de un estudio piloto en 21 pacien- tes de M.A. Merino y cols.}

El estudio de Merino y cols., vuelve a poner de manifiesto la complejidad subyacente a las alteraciones de la oxigenación y el metabolismo cerebral tras un traumatismo craneoencefálico. Desde la monitorización de la PIC,
35. Suistomaa, M., Ruokonen, E., Kari, A., Takala, J.: Time-pattern of lactate and lactate to pyruvate ratio in the first 24 hours of intensive care emergency admissions. Shock. 2000; 14: 8-12.

36. Tofteng, F., Larsen, F.S.: Monitoring extracellular concentrations of lactate, glutamate, and glycerol by in vivo microdialysis in the brain during liver transplantation in acute liver failure. Liver Transpl. 2002; 8: 302-305.

37. Ungerstedt, U., Rostami, E.: Microdialysis in neurointensive care. Curr Pharm Des. 2004; 10: 2145-2152.

38. Verweij, B.H., Muizelaar, J.P., Vinas, F.C., et al.: Impaired cerebral mitochondrial function after traumatic brain injury in humans. J Neurosurg. 2000; 93: 815-820.

39. Vespa, P., Bergsneider, M., Hattori, N., et al.: Metabolic crisis without brain ischemia is common after traumatic brain injury: a combined microdialysis and positron emission tomography study. J Cereb Blood Flow Metab. 2005; 25: 763774 .

40. Vespa, P., McArthur, D.L., Alger, J., et al.: Regional heterogeneity of post-traumatic brain metabolism as studied by microdialysis, magnetic resonance spectroscopy and positron emission tomography. Brain Pathol 2004; 14 : 210-214.

41. Werner, C., Engelhard, K.: Pathophysiology of traumatic brain injury. Br J Anaesth. 2007; 99: 4-9.

42. Xiong, Y., Gu, Q., Peterson, P.L., Muizelaar, J.P., Lee, C.P.: Mitochondrial dysfunction and calcium perturbation induced by traumatic brain injury. J Neurotrauma. 1997; 14: 23-34.

Merino, M.A.; Sahuquillo, J.; Borrull, A.; Poca M.A.; Riveiro, M.; Expósito, L.: ¿Es el lactato un buen indicador de hipoxia tisular? Resultados de un estudio piloto en 21 pacientes con un traumatismo craneoencefálico. Neurocirugía 2010; 21: 289-301.

Correspondencia: M. Ángeles Merino. Unidad de Investigación de Neurotraumatología y Neurocirugía. Hospital de Traumatología (Planta -2) del Hospital Universitario Vall d'Hebron. Paseo Vall d'Hebron 119-129. 08035, Barcelona, España. e-mail: mamerino@neurotrauma.net

hemos vivido un progresivo incremento de los dispositivos empleados en el control de los enfermos neurocríticos, de forma que cada uno de ellos nos proporciona una visión limitada de una parcela concreta de la fisiopatología de la 
enfermedad. Como suele ser habitual, el incremento de la información disponible no encaja en los viejos esquemas elaborados en el pasado sobre paradigmas relativamente simples. Por el contrario, la suma de más datos acerca del metabolismo energético tisular en el enfermo neurocrítico nos pone delante de escenarios de interpretación más compleja, aparentemente contradictorios pero seguramente más cercanos a la realidad.

El principal hallazgo de los autores es la constatación de la falta de concordancia entre datos que aparentemente deberían señalar un mismo fenómeno (niveles de lactato e índice lactato/piruvato), y que abundan en un papel distinto para el lactato del que clásicamente hemos aceptado, así como la importancia de considerar ambas moléculas (lactato y piruvato) para obtener una visión más real del equilibrio redox tisular. Se nos recuerda en la discusión que el papel de estas moléculas excede el de meros eslabones del metabolismo energético celular y que intervienen en otras vías (p. ej. reacciones anapleróticas del ciclo de Krebs) que podrían explicar en algunas ocasiones sus oscilaciones. La influencia de la propia situación sistémica o las alteraciones enzimáticas deben considerarse también a la hora de interpretar los datos obtenidos.

Por otro lado, la disociación existente entre la información que proporciona la $\mathrm{PtiO}_{2}$ y la microdiálisis vuelve a señalar que en el enfermo neurotraumático hoy en día, cuando la prevención de la lesión secundaria por alteraciones de la perfusión o la oxigenación cerebral cada vez está mejor controlada, las situaciones de viraje metabólico hacia la anaerobiosis en presencia de adecuados niveles de $\mathrm{O}_{2}$ tisular son cada vez más reconocibles. La relevancia clínica de elevaciones moderadas del lactato o el índice lactato-piruvato sobre los niveles considerados actualmente como normales, y un conocimiento más profundo de estos propios márgenes de normalidad en diversas circunstancias fisiológicas, son cuestiones todavía no respondidas completamente. Lo que si es cierto es que sin la posibilidad de determinar la química intersticial los estados de profundo trastorno metabólico sin hipoxia tisular detectable van a pasar desapercibidos, y la superación de estas anomalías, que son mucho más habituales de lo que se suponía, es probablemente una de las próximas fronteras a conquistar en el intento de mejorar el pronóstico de estos enfermos.

Desde un punto de vista crítico, cabría lamentar la selección, de entre una tan vasta fuente de información y casos posibles, de algunos pacientes incluidos en el análisis con sensores de $\mathrm{PtiO}_{2}$ y catéteres de microdiálisis posicionados en hemisferios contralaterales, pues aún en el caso de lesiones difusas, no podemos descartar la existencia de diferencias regionales, y a la hora de establecer comparaciones entre la información procedente de ambas fuentes, cabría plantear la cuestión de si en esos casos ambos sistemas proporcionan información verdaderamente congruente sobre un mismo proceso fisiopatológico.

En resumen, se trata de un estudio de objetivos sencillos pero bien establecidos y relevantes, informativo para los clínicos, adecuadamente planteado y ejecutado, y que, aunque no aporta información verdaderamente nueva, resalta conceptos recientes que resultarán novedosos para muchos lectores.

M.Brell

J.Ibáñez

Palma de Mallorca 